\title{
Maternal body composition in relation to twinning
}

\author{
Thomas Nilsen, Per Magnus and Ragnhild Ørstavik \\ Norwegian Institute of Public Health, Norway \\ Correspondence: Thomas Nilsen, Norwegian Institute of Public Health, P.O. Box 4404, 0403 Oslo, Norway \\ E-mail: thomassevenius.nilsen@fhi.no
}

\begin{abstract}
Monozygotic (MZ) twinning is considered to be a random event whereas spontaneous dizygotic (DZ) twinning is influenced by several factors. Thus, secular changes in twinning rates are usually explained by changes in DZ twinning alone. Maternal body mass index (BMI) before pregnancy and maternal height are believed to be significant drivers of twinning. Our aim in this study was to explore to what degree maternal body composition influences twinning. Data on births and maternal height and BMI from the Medical Birth Registry Norway (MBRN) was analyzed applying multivariate logistic regression analysis. The results showed that increasing maternal BMI and height has a positive association with twinning. There is an increased risk of DZ twinning for a maternal BMI $>25$, OR 1.31-1.43 and for maternal height $\geq 173 \mathrm{~cm}$, OR 1.28. In explaining secular changes in the twinning rate, one has to take into account secular changes in maternal body composition.
\end{abstract}

This is an open access article distributed under the Creative Commons Attribution Licence, which permits unrestricted use, distribution, and reproduction in any medium, provided the original work is properly cited.

\section{INTRODUCTION}

Monozygotic (MZ) twinning is considered to be a random event and the rate of monozygotic twinning is mostly stable at $1 / 3$ of twin births in western countries. Spontaneous dizygotic (DZ) twinning is influenced by several factors. Of these, the most important is the mother's age at conception $(1,2)$. Tandberg et al. (3) reported a 2.5 relative risk of having twins for women 38 years old compared to women 20 years old, based on data from the Medical Birth Registry of Norway (MBRN). However, it has also been recognized that maternal body composition has a significant effect on twinning. Several studies have shown an association between obesity (BMI $>30)$ and dizygotic twinning (4-6). These studies also confirm maternal height as an independent factor associated with twinning.

The correlation between age and twinning is believed to be caused by age related increase in levels of Follicle Stimulating Hormone (FSH). Increased FHS levels has also been linked to the relationship between twinning and maternal weight, as obesity is associated with hormonal changes (1). Through which biological process maternal height is associated with twinning is not known. One possibility is that twinning is linked to diet (7), and height being a marker of nutrition level will then be associated with twinning. Some evidence points towards the role of insulin growth factor (IGF) which is found in dietary products and meat. IGF is believed to enhance ovarian follicle recruitment and decrease ovum aptoptosis (7). There is also speculation that twinning is an adaptive natural selection process where lifetime reproductive success is maximized for mothers having twins in environments of abundant food availability - abundant food being associated with increasing height (2). An underlying motive for investigating possible drivers in twinning stems from the observation that there are significant changes in the secular twinning rate. In the western world twinning has increased noticeably in the last three decades, as noted in several studies $(3,8,9)$. Norway has seen a near doubling of the twinning rate since the start of MBRN in 1967 to the present day. In 1967, 1.01\% of all births where twin births, with a maximum in 2002 of $1.97 \%$, and a somewhat lower rate in 2012 of $1.73 \%$.

This trend may be due to delayed childbearing and the introduction of various assisted reproductive technologies (ART). The overall twinning rate has in later years somewhat abated due to changes in in vitro fertilization (IVF) practice with the introduction of elective single embryo transfer (eSET) (10). Excluding ART pregnancies, reported in MBRN from 1988, the twinning rate in 2012 is $1.43 \%$ - an increase of nearly $50 \%$ since 1967 . One study found that even after excluding ART and adjusting for maternal age for Norway, there was a significant increase in twinning rates from the period 1967-1987 to the period 1988-2004 for all age groups (3). This implies that other factors affecting the twinning rate are at play. As mentioned above, maternal body composition is shown to be significantly associated with DZ twinning. If this is the case, secular changes in maternal body composition in Norwegian women should be considered when explaining changes in the twinning rate. Previous Norwegian twinning studies have not been able to investigate the role of body mass index (BMI) and maternal height in twinning incidence as the mother's weight before pregnancy was not registered in MBRN until 2007.

The purpose of this study was to explore the role of maternal body composition in twinning, based on Norwegian registry data. To get a cursory idea of the magnitude of the maternal body composition effect on the current twinning rate, if any, we also examined the attributable fraction of BMI on twinning. 
Table 1. Summary statistics of MBRN sample.

\begin{tabular}{lccc}
\hline Years 2007-2012 & Total number of births & Twin births & ART twin births \\
\hline Number & 139379 & 2326 & 508 \\
Mean maternal age & 29.6 & 31.3 & 33.1 \\
Mean parity & 0.87 & 0.85 & 0.46 \\
Smoking before pregnancy & $17.6 \%$ & $17.6 \%$ & $12.0 \%$ \\
(irregularly \& daily) & & & \\
Mean (range) maternal BMI & $24.4(11.7-80.0)$ & $24.7(14.8-48.6)$ & $24.5(15.8-48.6)$ \\
Mean (range) maternal height, cm & $166.9(132-199)$ & $167.9(140-188)$ & $168.1(150-186)$ \\
\hline
\end{tabular}

\section{MATERIALS AND METHODS}

Information on pregnancies and births and maternal characteristics were collected from MBRN, which is a mandatory nationwide birth registry comprising all births in Norway since 1967 (11). Pregnancies ending after week 12 are notifiable to the MBRN, which thus includes live and still births, and induced abortions after the first trimester. MBRN also contains information on ART (in vitro fertilization and intracytoplasmic sperm injection).

Data on maternal height and weight (BMI) before pregnancy were collected from 2007 and onwards, and are currently only reported from around $40 \%$ of birth clinics.

Since the registry unit in MBRN is the child, pregnancies with a plurality of two, having a record for each child, were collapsed into one record, thus, counting each twin birth only once. Total number of births in MBRN for the study period (2007-2012) was 361,560. Of these only 139,468 had information on pre pregnancy BMI. Hence, of a total of 361,560 births 222,092 were excluded. Since we were interested in the contributing causes to twin conception all "births" were included regardless of outcome. Births with a higher plurality (greater than two) were excluded. Birth records where there was no ID for the mother, or births which pertained to pregnancies with a plurality $>1$ but had missing record for the other child were also excluded. One record was excluded due to obvious misreporting of height and weight. No other selection criteria were employed. The final sample comprised 139,379 births (years 2007-2012).

We applied logistic regression analyses to test the effect of BMI and height on twinning. First we modelled overall twinning (regardless of zygosity). As we assumed that only $\mathrm{DZ}$ twinning is influenced by BMI and height, and MBRN has no information on zygosity, a second model with only opposite sex twins was analyzed. The models were adjusted for maternal age, parity and smoking before pregnancy, which are believed to influence DZ twinning $(2,5)$. Subsequently, analyses were stratified for smoking categories ("not regularly smoking" versus "daily smoking"). Smoking is believed to affect the hormonal balance and increase the probability of double ovulation (12). Parity has been suggested to have an effect independent of maternal age, although the two are highly correlated (13).
Reference BMI was $18.5-25 \mathrm{~kg} / \mathrm{m}^{2}$. Height categories are according to those of Basso et al. (4) for comparison, and reference height was set to $165-168 \mathrm{~cm}$, which includes the sample mean height $(166.9 \mathrm{~cm})$. Stata 13SE was used for the analyses (14). The attributable fraction (denoted $\lambda$ ) for BMI was calculated using the formula $\lambda=P_{E}(R R-1) /\left[P_{E}(R R-1)+1\right]$, where $P_{E}$ is the proportion of exposed (maternal $\mathrm{BMI}>25$; reference group BMI 18.5-25) in the sample and RR is the relative risk of twinning compared to the nonexposed group (15).

\section{Results}

Mother's mean BMI before pregnancy was 24.4 (range 11.7-80.0) and mean height was 166.9 (range 132199) $\mathrm{cm}$. In Table 1 we give summary statistics for the sample on mean age, parity, twin births, ART, smoking, height and weight.

For all twin births BMI was weakly, but significantly associated with twinning (OR 1.01, $\mathrm{p}=0.004)$, when BMI was modelled as a continuous variable. However, when categorizing BMI, making the model more easily interpretable, regression outcomes, reported in Table 2 , showed that for all twin births, BMI $>25$ was not significantly associated with twinning. For BMI below normal $(<18.5)$, however, there was a decreased risk of twinning (OR 0.66). Height was significantly associated with twinning for maternal height $\geq 173 \mathrm{~cm}$ with increased odds of twinning (OR 1.22), and reduced risk for heights $<165 \mathrm{~cm}$ (OR 0.85). In an analysis restricted to opposite sexed DZ twins, increasing BMI was significantly associated with twinning. For BMI $25-30$ and $\mathrm{BMI} \geq 30$, the ORs were 1.31 and 1.43 , respectively. Low BMI $(<18.5)$, however, was not significantly associated with a reduced risk of twinning. For maternal height, height $\geq 173 \mathrm{~cm}$ was significantly positively associated (OR 1.28 ) and height $<165 \mathrm{~cm}$ negatively (OR 0.78 ) associated with $\mathrm{DZ}$ twinning. For the years 2007-2012 the attributable fraction was $12.0 \%$ (95\% CI $6.7-20.4 \%$ ), i.e. the fraction of twinning that could be attributed to a maternal BMI $>25$.

\section{DISCUSSION}

Our results show that maternal body composition is influencing the risk of twinning, especially DZ twinning. Furthermore, our results confirm findings from 
Table 2. ORs and 95\% CI for risk of twinning in each BMI and height category, adjusted for maternal age, parity and smoking.

\begin{tabular}{|c|c|c|c|}
\hline Variable & Singleton births & Twin births & Adjusted OR $(95 \% \mathrm{CI})^{* *}$ \\
\hline & \multicolumn{3}{|c|}{ All twins } \\
\hline \multicolumn{4}{|l|}{ BMI } \\
\hline$<18.5$ & 5454 & 45 & $0.66(0.48-0.89)^{*}$ \\
\hline $18.5-24.9$ & 81007 & 1073 & Ref \\
\hline $25-29.9$ & 29622 & 437 & $1.09(0.97-1.22)$ \\
\hline$\geq 30$ & 16027 & 249 & $1.14(0.99-1.31)$ \\
\hline \multicolumn{4}{|l|}{ Height, cm } \\
\hline$<165$ & 46584 & 527 & $0.85(0.75-0.97)^{*}$ \\
\hline $165-168$ & 32294 & 436 & Ref \\
\hline $169-172$ & 27856 & 412 & $1.07(0.94-1.23)$ \\
\hline \multirow[t]{2}{*}{$\geq 173$} & 25376 & 432 & $1.22(1.07-1.40)^{*}$ \\
\hline & \multicolumn{3}{|c|}{ Opposite sexed twins } \\
\hline \multicolumn{4}{|l|}{ BMI } \\
\hline$<18.5$ & 5454 & 18 & $1.05(0.65-1.70)$ \\
\hline $18.5-24.9$ & 81007 & 289 & Ref \\
\hline $25-29.9$ & 29622 & 141 & $1.31(1.07-1.60)^{*}$ \\
\hline$\geq 30$ & 16027 & 84 & $1.43(1.12-1.83)^{*}$ \\
\hline \multicolumn{4}{|l|}{ Height, cm } \\
\hline$<165$ & 46584 & 145 & $0.78(0.62-0.99)^{*}$ \\
\hline $165-168$ & 32294 & 134 & Ref \\
\hline $169-172$ & 27856 & 117 & $1.00(0.76-1.27)$ \\
\hline$\geq 173$ & 25376 & 139 & $1.28(1.00-1.62)^{*}$ \\
\hline
\end{tabular}

previous studies. A study from the Collaborative Perinatal Project in the United States (6) reported a statistical significant increase in risk of twinning with increasing BMI $(\mathrm{p}<0.001)$. For a BMI $\geq 30$ compared to the reference group $(\mathrm{BMI}<20)$ the adjusted odds ratio (OR) was 2.07 (95\% CI 1.30-3.29) for DZ twinning whereas $\mathrm{MZ}$ twinning was not significantly related to BMI. This estimate was somewhat higher than our results (OR 1.31-1.43), but the difference is probably due to choice of reference group, BMI $<20$. Findings very close to ours were reported in a Dutch study, which found an increase in spontaneous DZ twinning with increasing BMI, OR 1.3 (95\% CI 1.11.4) for overweight vs. normal weight women (5). Similar findings have also been reported in a Danish study based on the Danish National Birth Registry, with an OR of 1.62 (95\% CI 1.07-2.47) for BMI $\geq 30$ versus normal BMI for opposite sexed twins (4).

For height our ORs for increased risk of overall and DZ only twinning was significant only for heights $\geq 173 \mathrm{~cm}$ (OR 1.22 and 1.28). This is in line with Basso et al. (4), who found that risk of overall twinning increased with increasing maternal height, OR 1.36 (95\% CI 1.10-1.69) for heights $\geq 173 \mathrm{~cm}$ compared to maternal height $<165 \mathrm{~cm}$. Somewhat higher estimates were reported by Hoekstra et al. (5) with significant results for all heights $>170 \mathrm{~cm}$ compared to reference $(<165 \mathrm{~cm})$; the risk of DZ twinning was OR 1.3 (height $170-173 \mathrm{~cm}$ ) and 1.6 (height $174-195 \mathrm{~cm}$ ) and Reddy et al. (2005) with OR 1.66 (95\% CI 1.16-2.37) for heights $\geq 165 \mathrm{~cm}$ (reference $<157.5 \mathrm{~cm}$ ). Again, this is probably due to different categorization of height and choice of reference height.

The risk of DZ twinning seems to increase linearly with increasing height and BMI. Although speculative, could it be that the probability of having twins is related to body size in the sense that a larger body has more space and resources to carry through a twin pregnancy, which after all is more demanding of the mother (16)? The MBRN data, which this study is based on, only register birth outcome after week 12 of the pregnancy. Hence, what we really measure is body composition in relation to birth outcome and not body composition in relation to spontaneous conception of either DZ or MZ twins, of which we have no information. If so, there might be an increased tendency for a spontaneous fetal reduction or miscarriage in the first trimester for underweight mothers carrying twins. It is well documented that a large part of spontaneous twin conceptions result in singleton births or none through spontaneous fetal reduction or miscarriage - causes of which are unknown $(17,18)$. This has some resonance with the theory, mentioned above, that twinning is also part of an adaptive natural selection process associated with food availability and nutrition, but in this case linked to completion of a multiple pregnancy and not multiple ovulation. Results from other studies in this regard are inconclusive. Basso et al. (4) found that $\mathrm{BMI}<20$ was associated with lower risk of overall twinning. However, Hoekstra et al. (5) found no 
evidence of significant decreased risk of DZ twinning to $\mathrm{MZ}$ twinning for the same BMI category when compared to reference (BMI 20-25), but they did not investigate overall twinning to singletons. Reddy et al. (6) used $\mathrm{BMI}<20$ as reference but found only evidence of increased risk of DZ twinning for $\mathrm{BMI} \geq 30$.

Having established that maternal height and weight has an effect on twinning, the question arises whether this effect is strong enough to explain changes in the twinning rate. As mentioned in the introduction, there has been a rise in the twinning rate the last decades, all of which is not explained by maternal age alone, the strongest driver, or other factors. We know that the worldwide increase in prevalence of overweight and obesity is also present in Norway. For women between 20-29 and 30-39 years old, there has been an in excess of 50\% increase in overweight and a 3-4 fold increase in obesity in the period 1984-1986 to 2006-2008 (19). It is reasonable to assume that this increase also has had an effect on the twinning rate, and might account for some of the increase in twinning that has occurred in Norway since the $1980 \mathrm{~s}$. MBRN has no information on maternal pre pregnancy BMI prior to 2007 and thus a direct estimate of the effect of changing maternal $\mathrm{BMI}$ on the twinning rate is not possible for these two periods. Also, the time interval covered by our data, years 2007-2012, is too narrow to explore secular changes in the twinning rate as a function of changes in maternal BMI. However, for the period 1984-1986 we can, as a proxy, use the BMI distribution of women of fertile age from the HUNT1 study, a population based health survey conducted in the county of NordTrøndelag, Norway (20). Using these data for the period 1984-1986 with the relative risk of twinning from the MBRN, we estimated the attributable fraction to $9.6 \%(95 \%$ CI $5.2-17.1 \%)$. Our result of $12 \%(95 \% \mathrm{CI}$ $6.7-20.4 \%$ ), for the period 2007-2012, is an increase of 2.4 percentage points. Although the confidence intervals are wide and overlapping, this gives an indication that some of the increase in twinning during the last decades might in part be explained by the increased prevalence of overweight and obesity amongst women of fertile age. However, further studies with longer time series of maternal BMI is needed to test this assumption.

\section{Limitations}

This study is based on data from MBRN, which is complete for all births in Norway. Maternal weight before pregnancy was only reported from 2007 onwards, and only for around $40 \%$ of the birth clinics. To our knowledge there are no specific selection criteria accounting for this underreporting, and it has probably not introduced any bias which would influence the effect estimates. The confidence intervals are relatively wide, and with more complete reporting they would narrow and might change the interpretation of some of the results. However, the main conclusion that body composition is associated with twinning will not be affected.

Another limitation concerns the ability to discriminate between natural conceptions and conceptions by ART. There is an underreporting of ART treatments in MBRN, especially for treatments conducted outside Norway. This could lead to bias as not all ART pregnancies are excluded from analysis. Tandberg et al. (3) concluded that the scope of misclassification of ART pregnancies did not have a substantial influence on the results in their analysis of secular changes in the Norwegian twinning rates; results from their study showed that around 40 multiple pregnancies were underreported each year for the period 1999-2004. Another limitation is that we were not able to adjust for fertility enhancing treatments which are not recorded in the MBRN database. If these treatments are associated with some of our independent variables and the outcome, they could influence the effect estimate of the variable of interest. Finally, it should be noted that in the calculation of the attributable fraction, the RRs are unadjusted; thus the estimate should be considered as an upper limit of the attributable fraction.

\section{CONCLUSION}

We have shown that maternal body composition has an influence on the twinning rate. Twinning is positively associated with increasing height and BMI. Analysis of secular changes in the twinning rate must, in addition to other known factors which are affecting the twinning rate, also take into consideration secular changes in maternal body composition.

\section{REFERENCES}

1. Hall JG. Twinning. Lancet 2003;362(9385):735-43.

2. Hoekstra C, Zhao ZZ, Lambalk CB, Willemsen G, Martin NG, Boomsma DI, et al. Dizygotic twinning. Hum Reprod Update 2008;14(1):37-47.

3. Tandberg A, Bjørge T, Børdahl PE, Skjærven R. Increasing twinning rates in Norway, 1967-2004: the influence of maternal age and assisted reproductive technology (ART). Acta Obstet Gynecol Scand 2007;86(7):833-9.

4. Basso O, Nohr EA, Olsen J, Christensen K. Relationship of maternal body mass index and height to twinning. Obstet Gynecol 2005;106(2):411; author reply.

5. Hoekstra C, Willemsen G, van Beijsterveldt CE, Lambalk CB, Montgomery GW, Boomsma DI. Body composition, smoking, and spontaneous dizygotic twinning. Fertil Steril 2010;93(3):885-93. 
6. Reddy UM, Branum AM, Klebanoff MA. Relationship of maternal body mass index and height to twinning. Obstet Gynecol 2005;105(3):593-7.

7. Steinman G. Can the chance of having twins be modified by diet? Lancet 2006;367(9521):1461-2.

8. Ananth CV, Chauhan SP. Epidemiology of twinning in developed countries. Semin Perinatol 2012;36(3):156-61.

9. Imaizumi Y. Trends of twinning rates in ten countries, 1972-1996. Acta Genet Med Gemellol (Roma) 1997; 46(4):209-18.

10. Sunde A. Significant reduction of twins with single embryo transfer in IVF. Reprod Biomed Online 2007;15 Suppl 3:28-34.

11. Irgens LM. The Medical Birth Registry of Norway. Epidemiological research and surveillance throughout 30 years. Acta Obstet Gynecol Scand 2000;79(6):435-9.

12. Olsen J, Bonnelykke B, Nielsen J. Tobacco smoking and twinning. Acta Med Scand 1988;224(5):491-4.

13. Fellman J, Eriksson AW. On the standardisation of the twinning rate. Twin Res 2002;5(1):19-29.

14. 2013. S. Stata Statistical Software: Release 13.: StataCorp LP.; College Station, TX.

15. Eide GE, Gefeller O. Attributable Fractions. In: Veierød M, Lydersen S, Laake P, editors. Medical statistics in clinical and epidemiological research. Oslo, Norway: Gyldendal, 2012.

16. Conde-Agudelo A, Belizan JM, Lindmark G. Maternal morbidity and mortality associated with multiple gestations. Obstet Gynecol 2000;95(6 Pt 1):899-904.

17. Landy HJ, Keith LG. The vanishing twin: a review. Hum Reprod Update 1998;4(2):177-83.

18. Dickey RP, Taylor SN, Lu PY, Sartor BM, Storment JM, Rye PH, et al. Spontaneous reduction of multiple pregnancy: incidence and effect on outcome. Am J Obstet Gynecol 2002;186(1):77-83.

19. Midthjell K, Lee CM, Langhammer A, Krokstad S, Holmen TL, Hveem K, et al. Trends in overweight and obesity over 22 years in a large adult population: the HUNT Study, Norway. Clin Obes 2013;3(1-2):12-20.

20. Drøyvold WB, Nilsen TI, Krüger Ø, Holmen TL, Krokstad S, Midthjell K, et al. Change in height, weight and body mass index: Longitudinal data from the HUNT Study in Norway. Int J Obes 2006;30(6):935-9. 